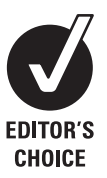
System, University of MissouriColumbia, Columbia, Missouri, USA

Correspondence to: S D Scott, Office of Clinical Effectiveness, University of Missouri Health System, One Hospital Drive, 1W-29, DC 103. 40, Columbia MO 65212, USA scotts@health.missouri.edu

Accepted 4 June 2009

\title{
The natural history of recovery for the healthcare provider "second victim" after adverse patient events
}

\author{
S D Scott, L E Hirschinger, K R Cox, M McCoig, J Brandt, L W Hall
}

\section{ABSTRACT}

Background: When patients experience unexpected events, some health professionals become "second victims". These care givers feel as though they have failed the patient, second guessing clinical skills, knowledge base and career choice. Although some information exists, a complete understanding of this phenomenon is essential to design and test supportive interventions that achieve a healthy recovery.

Methods: The purpose of this article is to report interview findings with 31 second victims. After institutional review board approval, second victim volunteers representing different professional groups were solicited for private, hourlong interviews. The semistructured interview covered demographics, participant recount of event, symptoms experienced and recommendations for improving institutional support. After interviews, transcripts were analyzed independently for themes, followed by group deliberation and reflective use with current victims.

Results: Participants experienced various symptoms that did not differ by sex or professional group. Our analysis identified six stages that delineate the natural history of the second victim phenomenon. These are (1) chaos and accident response, (2) intrusive reflections, (3) restoring personal integrity, (4) enduring the inquisition, (5) obtaining emotional first aid and (6) moving on. We defined the characteristics and typical questions second victims are desperate to have answered during these stages. Several reported that involvement in improvement work or patient safety advocacy helped them to once again enjoy their work. Conclusions: We now believe the post-event trajectory is largely predictable. Institutional programs could be developed to successfully screen at-risk professionals immediately after an event, and appropriate support could be deployed to expedite recovery and mitigate adverse career outcomes.

\section{BACKGROUND}

The Institute of Medicine report, To Err is Human, described staggering numbers of projected deaths each year as a result of preventable medical errors. ${ }^{1}$ Although these numbers are deeply disturbing, what accompanies each of these errors are countless health professionals closely involved in the event. Post-event investigations often reveal that experienced, wellintentioned staff are surrounded by complex clinical conditions, poorly designed processes and suboptimal communication patterns. These events leave a devastating personal and professional toll on staff.

During the mid-1980s, publications started appearing in the literature, which showcased numerous personal stories relaying intense feelings of incompetence, inadequacy or guilt after a medical error. $^{2-5}$ The personal stories were followed by accounts declaring a need for institutional support. ${ }^{6-12}$ The term second victim was initially coined by $\mathrm{Wu}^{13}$ in his description of the impact of errors on professionals. Others proposed that second victims experience post-traumatic stress disorder. ${ }^{14}$ Wolf et $a l^{15}$ described a unique, traumatic response by second victims in terms of emotional, social, cultural, spiritual and physical characteristics. A survey of more than 3000 physicians validated that, when involved in medical errors, emotional distress is prevalent and support was needed but was largely unaddressed. ${ }^{16}$ Crigger $^{17}$ described intense struggles given a traditional image of perfection among healthcare professionals. Human fallibility versus perfection is not deeply integrated within many health professional training programs, so preparation for medical error consequences is far from developed. ${ }^{18}$ Rossheim ${ }^{19}$ warns about excellent clinicians who may leave the profession prematurely when involved in a preventable error. Two decades after this issue was first described, White et a l2 $^{20}$ stressed the need for institutional commitment and support to address second victim needs and that most facilities probably had untapped internal resources. Denham ${ }^{21}$ proposed the formalization of second victim "rights" so that an automatic institutional response is stimulated.

The University of Missouri Health Care (UMHC) is an academic healthcare system in the Midwest that provides comprehensive healthcare services through primary care, inpatient acute care, longterm acute care and outpatient clinics. The Office of Clinical Effectiveness' (OCE) mission for UMHC is to transform the safety culture and manage the institutional response to preventable events and unexpected outcomes. During numerous event investigations, we became acutely aware of professional suffering and began to look for answers about this phenomenon and how to support our valuable care givers. To quantify the prevalence of the phenomenon at UMHC, two items were added to an internal patient safety culture survey ${ }^{22}$ assessment in May 2007. Almost one in seven staff (175/ $1160)$ reported they had experienced a patient safety event within the past year that caused personal problems such as anxiety, depression or concerns about the ability to perform one's job. Furthermore, $68 \%$ of these reported they did not receive institutional support to assist with this stress.

As a result of our internal findings and after studying support infrastructures from Critical Incident Stress Management techniques ${ }^{23}{ }^{24}$ and a formal support network for patients, families and 
clinicians known as Medically Induced Trauma Support Services, ${ }^{25}$ the OCE initiated a focused and deliberate set of actions and a research plan to gain a better understanding of what seemed to be somewhat predictable responses to the experience. A committee was commissioned to further explore care giver experiences and to define institutional support for what has been described as the darkest hour of one's professional career. The following consensus definition of second victims was reached to help drive identification of these individuals:

Second victims are healthcare providers who are involved in an unanticipated adverse patient event, in a medical error and/or a patient related injury and become victimized in the sense that the provider is traumatized by the event. Frequently, these individuals feel personally responsible for the patient outcome. Many feel as though they have failed the patient, second guessing their clinical skills and knowledge base.

This article describes and characterizes the information obtained through this qualitative exploratory study of the experiences and recovery trajectory of past second victims.

\section{METHODS}

After approval from the institutional review board for this unfunded study, the OCE identified professionals involved with patient safety event investigations between 2003 and 2007. A 25 -item semistructured interview guide was developed and included personal and professional demographics, participant recount of adverse event circumstances, physical/psychosocial symptoms experienced and recommendations for improving post-event support (table 1).

After a planning meeting where the protocol was delineated, the four-person interview team, consisting of two safety/risk management experts, one certified holistic nurse, and one sociologist, determined an interview schedule. The goal was to complete 30 interviews with professionals representing physicians, nurses and other disciplines. Subjects were contacted by phone with a description of the study. For those willing to participate, a one-hour face-to-face interview was agreed upon in a private location. Participants were assured anonymity and confidentiality. Before the interview, the consent form was reviewed and signed. Interviews were tape-recorded.

Upon conclusion of each interview, recordings were assigned a subject number and identified only by professional type. Tapes were transcribed by one person with another verifying deidentification and transcription accuracy. The interviewers initially conducted independent and iterative readings of all transcripts to identify patterns, experience characteristics and progression. This group then met regularly until themes were negotiated by focusing on experiential commonalities from the moment of the event through the transcribed interview experience. The themes were elucidated into stages and characteristics as the focus of the analysis combined experience trajectories with emotional support desired versus received. The stages and their characteristics were then presented to a volunteer interprofessional interest group who initially offered group critique but requested additional time to apply the work to actual real-time cases. The group reconvened after 1 month to finalize terminology used to describe and further differentiate the stages and characteristics.

\section{RESULTS}

Forty-three were contacted for study enrolment with 38 agreeing to participate and 31 who completed interviews. Reasons for declining participation included workload constraints $(n=2)$, maternity leave $(n=2)$ and a desire not to revisit the event $(n=1)$. Six were unable to schedule interviews and two who initially agreed opted to decline participation after informed consent was reviewed. One nurse learned about the research project and requested study enrolment (table 2).

Eighteen of the participants (58\%) were women; 10 were physicians, 11 registered nurses and 10 other health professionals (table 3). The duration of professional experience ranged between 6 months and 36 years (mean 13.5 years). Time since the adverse event ranged from 3 weeks to 44 months (mean 14 months).

Regardless of sex, professional type or years in the profession, the second victim phenomenon can be described as a life-altering experience that left a permanent imprint on the individual. Participants were able to provide meticulously detailed accounts of the event, some citing the exact date of the event even years later. Seventeen psychosocial and six physical symptoms were reported 10 or more times by the 31 participants (table 4).

Several factors seem to influence the intensity of the experience such as the relationship between the patient and care giver, past clinical experiences, a patient the same age as a family member or any other perceived "connection".

I remember feeling horribly sad that I couldn't do more for this child. This hit me harder than most of them. For some reason I really related with this family-I guess one reason is that the child was the age of my oldest daughter and I guess that I felt that this could have been my family. They were a nice family and didn't deserve to have this outcome. I cried a lot over this case and I guess I still cry when I think about her.

Many participants relived the event when triggered by an external stimulus. Different triggers were described such as a different patient in the exact location as the original event, a similar name, a similar diagnosis or a similar clinical situation. With a trigger, participants experienced similar physical or psychological symptoms as they did when the event initially unfolded:

No matter how much you fool yourself you are over something, and maybe even though I hadn't thought of it for months, I had that woman's name seared into my memory and as soon as I saw that name, my chest was up in my throat.

I still think about it. Just randomly you forget and then something will happen and it just pops into your head. You go over it again, what could I have done differently, what could I have said, what should I have done?

\section{Stages of recovery}

Participants developed their own unique way of coping, yet each described a predictable recovery trajectory. During iterative analyses, six stages emerged to describe this recovery trajectory. As outlined in table 5, stages were identified and named as follows: (1) chaos and accident response, (2) intrusive reflections, (3) restoring personal integrity, (4) enduring the inquisition, (5) obtaining emotional first aid and (6) moving on.

The first three stages occur after "impact realization" and were unique in that the second victim may pass through one or more of these stages simultaneously.

\section{Stage 1: Chaos and accident response}

At the moment that an adverse event/outcome is detected, the involved clinician described chaotic and confusing scenarios of both external and internal turmoil that ultimately led to a realization about what had occurred. During the immediate aftermath, there is a period of rapid inquiry to verify exactly what happened. Simultaneously, the patient might be unstable 
Table 1 Second victim interview guide

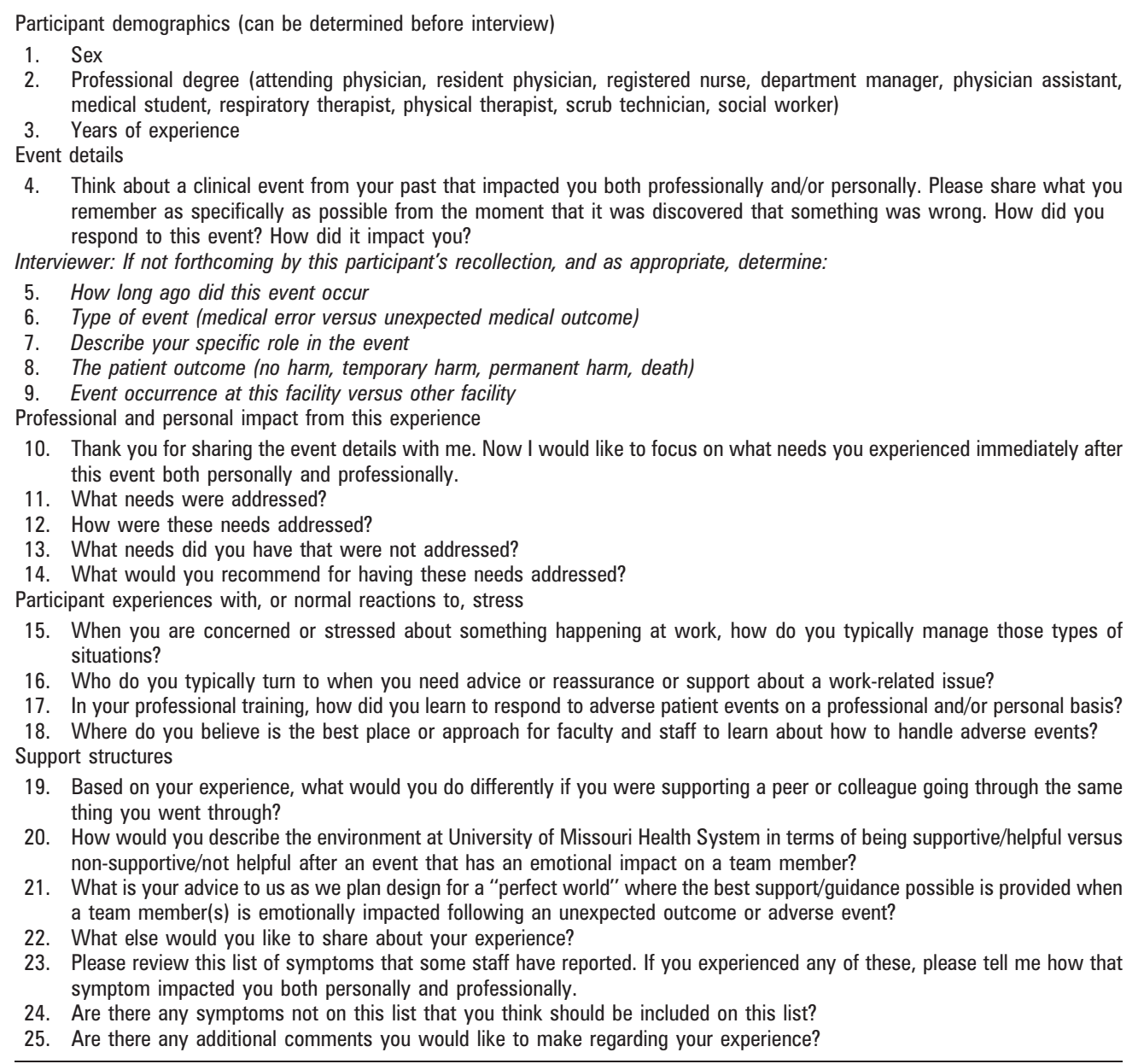

and require intensive care and monitoring. Usually, additional clinicians are summoned to provide support with procedures or testing. The victim is frequently distracted, immersed in self-reflection, while also trying to manage a patient in crisis. They often engaged a peer for patient care and chastised themselves for not being able to think coherently.

Right after the event and during the code, I was having trouble concentrating. It was nice to have people take over, that knew what they were doing, that I trusted. I was in so much shock. I don't think I was as useful as I usually am during a code situation.

\section{Stage 2: Intrusive reflections}

This stage was described as a period of haunted re-enactments, often with feelings of internal inadequacy and periods of self-isolation. The victim re-evaluates the situation repeatedly with "what if" questions.

I started to doubt myself. This shouldn't have happened. It was all hindsight but I kind of kept thinking over and over again.

There were some things that I thought maybe if I'd have done it this way, it wouldn't have happened or been avoided. Everything was clearer looking at things in retrospect. I lost my confidence for some time.

\section{Stage 3: Restoring personal integrity}

The third stage was described as seeking support from an individual with whom they had a trusting relationship such as a colleague, supervisor, personal friend or family member. Many did not know to whom they should turn because no one could

Table 2 Interview participation by professional groups

\begin{tabular}{llll}
\hline & $\begin{array}{l}\text { Number of } \\
\text { potential } \\
\text { subjects } \\
\text { approached for } \\
\text { participation }\end{array}$ & $\begin{array}{l}\text { Number of subjects } \\
\text { who agreed to } \\
\text { participate }\end{array}$ & $\begin{array}{l}\text { Number of subjects } \\
\text { who completed } \\
\text { interview process }\end{array}$ \\
\hline Professional type & 12 & 12 & 10 \\
Physician & 17 & 14 & 11 \\
Registered nurse* $^{*}$ & 14 & 12 & 10 \\
Other & 43 & 38 & 31 \\
\hline Total & 43 & & 12 \\
\hline
\end{tabular}

${ }^{*}$ One registered nurse contacted the research team and requested to be included.

†Managers, physician assistant, medical student, respiratory therapist, scrub technician, social worker, and physical therapist. 
Table 3 Participant demographics

\begin{tabular}{lllll}
\hline Years of experience & Physician & Registered nurse & Other & Total \\
\hline $0-5$ & 6 & 2 & 1 & 9 \\
$6-10$ & 2 & 2 & 2 & 6 \\
$11-15$ & 1 & 2 & 0 & 3 \\
$16-20$ & 0 & 3 & 3 & 6 \\
$21-25$ & 1 & 2 & 1 & 4 \\
$26-30$ & 0 & 0 & 2 & 2 \\
$31-36$ & 0 & 1 & 0 & 1 \\
Range & $1-25$ & $0.5-36$ & $6-30$ & \\
Mean years of experience & 7.3 & 15.2 & 17.7 & 13.5 \\
\hline
\end{tabular}

relate to their experience or understand the personal impact the event had on them professionally and personally. A consuming doubt regarding their future professional career plagued many. One of the biggest challenges was getting through personal reflections such as "what will others think of me" and "will I ever be trusted again":

\footnotetext{
I thought every single day for months I'd walk in and think everyone knows what happened because that's what happens in a unit where everyone works closely. I thought, "Do they think of me everyday as this loser who doesn't know what is going on?"

I thought, "These people are never going to trust me again".
}

Second victims described an inability to move forward when the event was followed by non-supportive, negative departmental "grapevine gossip", which triggered additional memories and intensified the self-doubt and lack of clinical confidence. The extent of this phase may well be anchored within a department's teamwork culture.

When I came back to work, no one really talked to me about it. For the most part, employees slowly found out about it from day to day. Nobody said anything about it and the next day, 2 people heard about it and somebody made fun of you or made some comment about you. Then a couple of days would go by and then someone else would find out about it. It took forever-I wanted to stand up in a staff meeting and say I did this because it would never end. I haven't heard anything about it for awhile from other employees but it took forever for it to trickle down the grapevine. That was frustrating.

\section{Stage 4: Enduring the inquisition}

After initial focus on stabilizing the patient and the personal reflections, there is an awakening that the institution will be reacting to the event in unclear ways. Specifically, the second victim starts to wonder about repercussions affecting job security, licensure and future litigation.

When this first happened, I was out of school for about 2 years. I thought my career was over. I assumed I would not [be in this profession] anymore. I thought I was going to get fired. I remember having to write an [incident report] about it, talk to the family and get my supervisor involved and it just became a whole days worth of work. I was totally exhausted. I was totally drained and thought I was going to lose my job. I have this degree and I will never be able to use it again.

The requirements of the Health Insurance Portability and Accountability Act (HIPAA) were poorly understood with regard to reviewing and/or discussing the case for support and healing.

It is really hard with HIPAA. With that situation there was nobody I could tell, not even my husband. All I could say is I have had a really horrible day. Because of HIPAA laws and our own professional values of confidentiality, we cannot take it home, other than to say I had a patient die today but not about the particular incident because it was too high profile.

\section{Stage 5: Obtaining emotional first aid}

Second victims tended to seek emotional support in a variety of ways. Many expressed concerns about not knowing who was a

Table 4 Most commonly reported physical and psychosocial symptoms

\begin{tabular}{llll}
\hline Physical symptoms & $\mathbf{n}(\%)$ & Psychosocial symptoms & $\mathbf{n}(\%)$ \\
\hline Extreme fatigue & $16(52)$ & Frustration & $24(77)$ \\
Sleep disturbances & $14(45)$ & Decreased job satisfaction & $22(71)$ \\
Rapid heart rate & $13(42)$ & Anger & $21(68)$ \\
Increased blood pressure & $13(42)$ & Extreme sadness & $21(68)$ \\
Muscle tension & $12(39)$ & Difficulty concentrating & $20(65)$ \\
Rapid breathing & $11(35)$ & Flashbacks & $20(65)$ \\
& & Loss of confidence & $20(65)$ \\
& & Grief & $20(65)$ \\
& & Remorse & $19(61)$ \\
& & Depression & $17(55)$ \\
& & Repetitive/intrusive memories & $16(52)$ \\
& & Self-doubt & $16(52)$ \\
& & Return to work anxiety & $15(48)$ \\
& & Second guessing career & $12(39)$ \\
& & Fear of reputation damage & $12(39)$ \\
& & Excessive excitability & $11(35)$ \\
\end{tabular}


Table 5 Research team consensus for trajectory of recovery

\begin{tabular}{|c|c|c|}
\hline & Stage characteristics & Common questions \\
\hline Stage 1 & Error realized/event recognized & How did that happen? \\
\hline \multirow{4}{*}{$\begin{array}{l}\text { Chaos and accident } \\
\text { response }\end{array}$} & Tell someone $\rightarrow$ get help & Why did that happen? \\
\hline & Stabilize/treat patient & \\
\hline & May not be able to continue care of patient & \\
\hline & Distracted & \\
\hline Stage 2 & Re-evaluate scenario & What did I miss? \\
\hline \multirow[t]{3}{*}{ Intrusive reflections } & Self isolate & Could this have been prevented? \\
\hline & Haunted re-enactments of event & \\
\hline & Feelings of internal inadequacy & \\
\hline Stage 3 & Acceptance among work/social structure & What will others think? \\
\hline \multirow[t]{3}{*}{ Restoring personal integrity } & Managing gossip/grapevine & Will I ever be trusted again? \\
\hline & Fear is prevalent & How much trouble am I in? \\
\hline & & How come I can't concentrate? \\
\hline Stage 4 & Realization of level of seriousness & How do I document? \\
\hline \multirow[t]{5}{*}{ enduring the inquisition } & Reiterate case scenario & What happens next? \\
\hline & Respond to multiple "why's" about the event & Who can I talk to? \\
\hline & Interact with many different "event" responders & Will I lose my job/license? \\
\hline & Understanding event disclosure to patient/family & How much trouble am I in? \\
\hline & Physical and psychosocial symptoms & \\
\hline Stage 5 & Seek personal/professional support & Why did I respond in this manner? \\
\hline \multirow[t]{3}{*}{ Obtaining emotional first aid } & Getting/receiving help/support & What is wrong with me? \\
\hline & Litigation concerns emerge & Do I need help? \\
\hline & & Where can I turn for help? \\
\hline Stage 6 & Dropping out & Is this the profession I should be in? \\
\hline \multirow{11}{*}{$\begin{array}{l}\text { Moving on (one of three } \\
\text { trajectories chosen) }\end{array}$} & 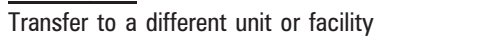 & Can I handle this kind of work? \\
\hline & Consider quitting & \\
\hline & Feelings of inadequacy & \\
\hline & Surviving & $\begin{array}{l}\text { How could I have prevented this from } \\
\text { happening? }\end{array}$ \\
\hline & Coping, but still have intrusive thoughts & Why do I still feel so badly/guilty? \\
\hline & Persistent sadness, trying to learn from event & \\
\hline & Thriving & What can I do to improve our patient safety? \\
\hline & Maintain life/work balance & What can I learn from this? \\
\hline & Gain insight/perspective & What can I do to make it better? \\
\hline & Does not base practice/work on one event & \\
\hline & Advocates for patient safety initiatives & \\
\hline
\end{tabular}

"safe" person to confide in. Approximately one-third of second victims sought support from loved ones but indicated that they were cautious when doing this because of above-mentioned privacy and legal considerations. Others noted that their loved ones just could not comprehend their professional life and should be protected from this hurt. A number of respondents did receive support from coworkers, supervisors or department chairs. However, the amount provided may be insufficient as negative feelings lingered.

After the event, I met with my attending who was specifically involved in the case and that helped a lot. But it was kind of funny, that was the first week and then it was turned off like a switch. It helped at first but I felt it just got dropped and that was a disappointment. Nobody wanted to talk about it. I am not a touchy, feely person but I at least needed someone to make sure I was doing okay and I never felt like that. I felt like, "Well, this happens and you should be better about it and that's it." There isn't a single day that this doesn't affect me.

Some felt that knowing where to go for support and what could be said was never made clear.

I didn't know what to do or who you were supposed to talk to professionally, legally. I didn't know how much I was allowed to say, where I should document things. We have policies for everything. We have [policies] if people get a needle stick, but if this happens, you don't know who to call. It needs to be a procedure like if you're a nurse, call your nurse manager. If you're an attending physician, you should sit down with your team and debrief. Let everyone discuss how they feel about what happened. I don't think any of those things are clear. I didn't know what to do.

Even attempts to provide formal professional support can fall short.

More access to [Employee Assistance Program] would be helpful. I felt like they wanted to assess where I was and say, "Okay we think you could benefit from more therapy. Here are some local doctors you could go to." If it is an employee assistance program, it should assist employees, instead of saying here are some local doctors, they should say, "We would like to continue doing some sessions with you before you go to that next level".

\section{Stage 6: Moving on-dropping out, surviving or thriving}

Although numerous second victims described their event as impacting their work practices, some felt the event would stay with them throughout their careers. There is a push internally 
(from the second victim) and externally (from coworkers, colleagues, supervisors) to "move on" and put the event behind them. However, participants found it difficult to completely put the event behind them. This is a unique stage for recovery as it has three potential paths: dropping out, surviving or thriving. Dropping out involved changing professional role, leaving the profession or moving to a different practice location. Perhaps the intensity of the haunted re-enactments drove these victims to second guess their professional abilities and drop out.

Overall, I didn't feel it was a good environment to stay in, in terms of healing is why I chose to leave. But in the new unit, it was very helpful.

I moved over to another service. I think a fresh start was good for me. It was devastating during that period.

It affected me greatly and made me question my abilities. Was I ready to be an attending?

Another possible conclusion in the sixth stage is surviving, which means the individual performs at the expected performance levels and is "doing okay" but continues to be plagued by the event.

\section{I figured out how to cope and how to say yes, I made a mistake. And that mistake caused a bad patient outcome but I haven't figured out how to forgive myself for that yet or to forget it. It's impossible to let go.}

Some retained the memories of the event, changed how they practiced or became involved in practice change. Thriving in the sixth stage was identified from participants who made something good come from the unfortunate clinic experience.

I couldn't really avoid getting back in the ambulance so what I did do was actually get back in the ambulance before the end of my shift and did a test ride to try to figure out why I was having so much trouble with the BP readout. I definitely needed to figure out a way for some good to come out of this horrible experience. I was questioning myself over and over again about what happened to me but then I thought, you know what, I've just had this experience in my life where I had to encounter this tragedy but it made me a better person. It really did, and it gave me more insight.

\section{CONCLUSION}

Regardless of sex, professional background or years of experience, all participants in our study easily recalled the immediate and ongoing impact of their specific career jolting event. Collectively, their emotionally charged accounts revealed a largely predictable recovery trajectory. Our analysis led to the identification and naming of six stages of recovery and stage characteristics. These were (1) chaos and accident response, (2) intrusive reflections, (3) restoring personal integrity, (4) enduring the inquisition, (5) obtaining emotional first aid and (6) moving on. The sixth stage, moving on, led to one of three outcomes: dropping out, surviving or thriving.

Participants provided insight into the type and quality of peer and institutional support they both received and desired. We believe frontline supervisors and peers could be trained to provide immediate and targeted support especially during the early stages. For the later stages, we believe most institutions have resources currently trained to provide emotional support for other critical incident situations. Examples include risk managers, chaplains, social workers, holistic or mental health clinicians, child life therapists and palliative care practitioners.

The first step, however, is the need to formulate an institutional awareness campaign that promotes open dialogue about the definition and prevalence of second victims. To quantify the prevalence locally, we recommend asking two questions: "Have you experienced a patient safety event within the past year that caused personal problems such as anxiety, depression, or concerns about your ability to perform your job?" and "If so, did you receive institutional support to assist you with this stress?" Every day, our healthcare professionals practice their art and science within enormously complex environments and experience unexpected patient outcomes. Many within healthcare systems suffer alone after events. It is imperative that an improved understanding of effective and immediate surveillance and support strategies be developed to mitigate the suffering among second victims.

Acknowledgements: The authors would like to recognize the dedication, contributions and deliberations of the University of Missouri forYOU Team (our "Second Victim" Steering Group) in advancing our understanding of this phenomenon and of Dr Jane Armer at the University of Missouri Sinclair School of Nursing for her scholarly guidance during our journey to systematically study this phenomenon.

Competing interests: None.

\section{REFERENCES}

1. Corrigan JM, Donaldson MS, Kohn LT, McKay T, Pike KC, Committee on Quality of Health Care in America. To err is human: building a safer health system. Washington, DC: National Academy Press, 2000.

2. Hilfiker D. Facing our mistakes. N Engl J Med 1984;310:118-22.

3. Levinson W, Dunn PM. A piece of my mind. Coping with fallibility. JAMA 1989;261:2252.

4. Hilfiker D. Healing the wounds: a physician looks at his work. New York, NY: Pantheon, 1985.

5. The mistake I'll never forget. Nursing90 1990;20:50-1.

6. Wu AW, Folkman S, McPhee SJ, et al. Do house officers learn from their mistakes? JAMA 1991;265:2089-94.

7. Christensen JF, Levinson W, Dunn PM. The heart of darkness: the impact of perceived mistakes on physicians. J Gen Intern Med 1992; 7:424-31.

8. Wu AW, Folkman S, McPhee SJ, et al. How house officers cope with their mistakes. West J Med 1993;159:565-9.

9. Newman MC. The emotional impact of mistakes on family physicians. Arch Fam Med 1996:5:71-5.

10. Wolf ZR, Serembus JF, Smetzer J, et al. Responses and concerns of healthcare providers to medication errors. Clin Nurse Spec 2000;14:278-87.

11. Engel KG, Rosenthal M, Sutcliffe KM. Residents' responses to medical error: coping, learning, and change. Acad Med 2006;81:86-93.

12. West CP, Huschka MM, Novotny PJ, et al. Association of perceived medical errors with resident distress and empathy: a prospective longitudinal study. JAMA 2006;296:1071-8.

13. Wu AW. Medical error: the second victim. The doctor who makes the mistake needs help too. BMJ 2000;320:726-7.

14. Rassin M, Kanti T, Silner D. Chronology of medication errors by nurses: accumulation of stresses and PTSD symptoms. Issues Ment Health Nurs 2005;26:873-6.

15. Wolf ZR. Stress management in response to practice errors: critical events in professional practice. PA-PSRS Patient Safety Advisory 2005;2:1-4.

16. Waterman AD, Garbutt J, Hazel E, et al. The emotional impact of medical errors on practicing physicians in the United States and Canada. Jt Comm J Qual Patient Saf 2007;33:467-76.

17. Crigger NJ. Always having to say you're sorry: an ethical response to making mistakes in professional practice. Nurs Ethics 2004;11:568-76.

18. Goldberg RM, Kuhn G, Andrew LB, et al. Coping with medical mistakes and errors in judgment. Ann Emerg Med 2002;39:287-92.

19. Rossheim J. To err is human - even for medical workers. Healthcare monster. http://healthcare.monster.ca/8099_en-CA_pf.asp (accessed 21 Jan 2009)

20. White AA, Waterman A, McCotter P, et al. Supporting health care workers after medical error: considerations for healthcare leaders. J Clin Outcomes Manag 2008;15:240-7.

21. Denham CR. Trust: the 5 rights of the second victim. J Patient Saf 2007;3 107-19.

22. Agency for Healthcare Research and Quality. Patient safety culture surveys. agency for healthcare research and quality. http://www.ahrq.gov/qual/hospculture/ (accessed 21 Jan 2009)

23. International Critical Incident Stress Foundation, Inc. http://www.icisf.org/ (accessed 21 Apr 2009).

24. Mitchell JT, Everly GS. Critical incident stress debriefing: an operations manual for CISD, defusing and other group crisis intervention services. 3rd edn. Ellicott City, MD: Chevron Publishing, 2001.

25. Medically Induced Trauma Support Services (MITSS). http://www.mitss.org/ (accessed 21 Apr 2009). 